\title{
Connectivity of Mobile Ad Hoc Sensor Networks for Vehicles
}

\author{
LI Shu ${ }^{\ddagger}$ \\ Department of Computer Science \\ and Technology, Tongji University \\ Shanghai, 201804,China \\ ailsa_leenew@sina.com
}

\author{
ZHANG Dong-Liang\$ \\ Department of Computer Science \\ and Technology, Tongji University \\ Shanghai, 201804,China \\ fire_zdl@163.com
}

\author{
JIANG Chang-Jun $\pi$ \\ Department of Computer Science \\ and Technology, Tongji University \\ Shanghai, 201804,China \\ cjjiang@online.sh.cn
}

\begin{abstract}
Reasonable network models are abstracted from the mobile ad hoc sensor network for the real road traffic system, and the connectivity of one-dimension (1-D) and two-dimension (2-D) networks are discussed separately by percolation theory. In the twodimension (2-D) network, a novel connectivity strategy, the pointbased coverage strategy, is introduced. Then through simulation experiments reasonable transmission radiuses of wireless nodes in real traffic network are obtained.
\end{abstract}

\section{Categories and Subject Descriptors}

G.3 [PROBABILITY AND STATISTICS] : Distribution functions, Experimental design, Probabilistic algorithms

\section{General Terms}

Performance, Design, Experimentation.

\section{Keywords}

percolation theory; topology control; ITS

\section{INTRODUCTION}

Intelligent Transportation Systems (ITS) combine many advanced technologies including information, communication and sensor to facilitate the transportation management. Sensors have become the "nerve ending" of vehicles, and users can obtain more information about roads through the Inter-Vehicles Communication (IVC), such as projects of CarTALK and FleetNet in EU, PATH in USA and DEMO2000 in Japan. In our ad hoc sensor network for ITS, there is only a vague idea of the number of nodes and the shape/size of the deployment region, but the expected node density is approximately known. Under the situation, percolation is the best research model. Percolation theory was introduced by BroadBent et al. as a mathematical model to study the flow of fluid in random media. Dousse et al. [1][2] analyzed the connectivity of ad hoc/sensor networks, and found the critical value of $\lambda$. In this paper, we use dynamic percolation theory to research the connectivity of our networks. The paper is structured as follows. Models are presented in Section 2. In Section 3, we study

Permission to make digital or hard copies of all or part of this work for personal or classroom use is granted without fee provided that copies are not made or distributed for profit or commercial advantage and that copies bear this notice and the full citation on the first page. To copy otherwise, or republish, to post on servers or to redistribute to lists, requires prior specific permission and/or a fee.

INFOSCALE 2007, June 6-8, Suzhou, China Copyright (๐) 2007 ICST 978-1-59593-757-5

DOI 10.4108/infoscale.2007.210 the characteristic of connecting in 1-D and 2-D dynamic random networks, and introduce a novel coverage strategy, the pointbased coverage strategy. Section 4 presents simulations and concludes of the connectivity of road vehicular network for Shanghai on our simulation platform.

\section{MODELS}

On the traffic map of Shanghai, we notice that national roads and provincial roads have less turnoff and are not congested, and vehicles on these roads drive straight for a long time. These roads have an almost 1-D shape (See Fig.1(a)). In downtown areas, roads are dense and these regions present a quite random traffic of 2-D plane (See Fig.1(b)). The vehicle traffic has randomicity and follows the Poisson distribution. So we abstract the network for vehicles as 1-D or 2-D dynamic random network. Assume that the wireless nodes are scattered according to a Poisson Point Process (P.P.P) on the space $\mathrm{R}^{d}$, where $d$ denotes the dimension. We use the Boolean Model [3] as the connectivity model. Simplify that the nodes move according to i.i.d. processes, so as to be benefit for our theoretic analysis. Our simulation platform is the Intelligent Transportation Service Grid System V3.0 designed by Department of Computer Science and Technology, Tongji University.

\section{CONNECTIVITY}

\subsection{1-D Dynamic random network}

Theorem 1: The probability that there exists a "gap" between two consecutive nodes in 1-D dynamic random network is strictly greater than zero.

Proof: Defining $\varphi$ to be the event that a "gap" appears between two consecutive nodes in 1-D static extended random networks. The probability that event $\varphi$ occurs in 1-D static network is one. In [2], given the initial node placement and the random mobility model, at any time $t$, the locations of the sensors still form a P.P.P of the same density. Therefore, the fraction of the area covered at time $t$ remains the same as in the initial configuration. So $P_{\lambda}(\varphi$ occurs for any time $t)=1$. The mobility does not affect the occurrence of $\varphi$. The theorem is proved, i.e. the full connectivity cannot occur a.s. in 1-D dynamic random network.

\subsection{Point-Based Coverage Strategy}

We introduce a novel definition of connecting in 2-D networks: point-based coverage strategy, which is suitable for car networks.

Definition 1: Given a static node $S$ on $\mathrm{R}^{2}$, let $\mathrm{D}$ be a square of size $[0, \sqrt{n}] \times[0, \sqrt{n}]$ and centered of $S$. All mobile nodes are distributed according to a P.P.P. at $\mathrm{t}=0$ and move i.i.d. For each 
mobile node $\mathrm{X}$ in area $\mathrm{D}$, node $\mathrm{X}$ is called covered by $S$ if there exists at least a path from the mobile node X to the static node $S$ in time $t$; and area $\mathrm{D}$ is called covered by $S$ if all mobile nodes in D have been covered by $S$ during time interval $[0, \mathrm{~T}]$.

We discuss the property of point-based coverage by percolation theory. We map our network model to a bond percolation model as [1](see Fig.2). The average number of nodes is equal to $n$ in extended network. The number of cells in $\mathrm{D}$ is $\frac{\sqrt{n} \times \sqrt{n}}{d^{2}}=\frac{n}{d^{2}}$. Then the probability that a cell contains at least one node is $p=1-\left(1-\frac{d^{2}}{n}\right)^{n}$, i.e. the probability that an edge is open is $p$.

Theorem 2: At any time $t$, if $p>\frac{16+2 \sqrt{10}}{27}$, there exists a.s. an open path from each mobile node $\mathrm{M}$ to the static node $S$ in D.

Proof: From the percolation theory in [7] we have:

$P\left(\exists\right.$ at least an open path starting at $\mathrm{S}$ to $\mathrm{M}$ in $\left.\mathrm{L}^{\prime}\right)$

$=1-P\left(\right.$ there exists no open path starting at $\mathrm{S}$ to $\mathrm{M}$ in $\left.\mathrm{L}^{\prime}\right)$

$=1-P(\exists$ a closed circuit surrounding $\mathrm{M}$ in $\mathrm{L})$

The number $\rho(l)$ of circuits of length $l$ in $\mathrm{L}$ is bounded by $\rho(l) \leq 4 \cdot 3^{l-2} \cdot l$. Denote $\sigma(l)$ the number of closed circuits of length $l$ in $\mathrm{L}$. The probability for a path of length $l$ to be closed is $q^{l}$. Then $\mathrm{P}(\sigma(l) \geq 1) \leq 4 \cdot 3^{l-2} \cdot l \cdot q^{l}$. The length of the closed circuit can be any value, so

$$
P(\sigma(l) \geq 1 \text { for any } l)=\sum_{l=1}^{\infty} P(\sigma(l) \geq 1) \leq \frac{4}{3} q \sum_{l=1}^{\infty} l(3 q)^{l-1}
$$

When $q<\frac{1}{3}, P(\sigma(l) \geq 1$ for any $l) \leq \frac{4 q}{3(1-3 q)^{2}}$. Let the above expression be equal to 1 , and we obtain $q<\frac{11-2 \sqrt{10}}{27}$. And by ergodicity, the theorem holds.

\section{SIMULATION AND CONCLUSION}

We define the fraction of connectivity in a simulation region as following: $p=\frac{\max \{\text { the number of nodes in each cluster }\}}{\text { the total number of nodes in network }}$.

The results of simulation show two factors affect the fraction of connectivity: the density of vehicles $\lambda$ and the transmission radius. The results in Fig.3(a) show that full connectivity may occur in
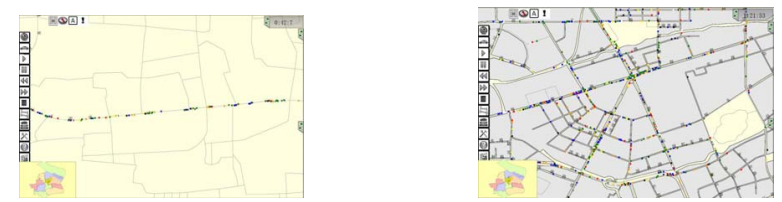

Fig.1(a)NO. 312 National Road (b)simulation of Wujiaochang
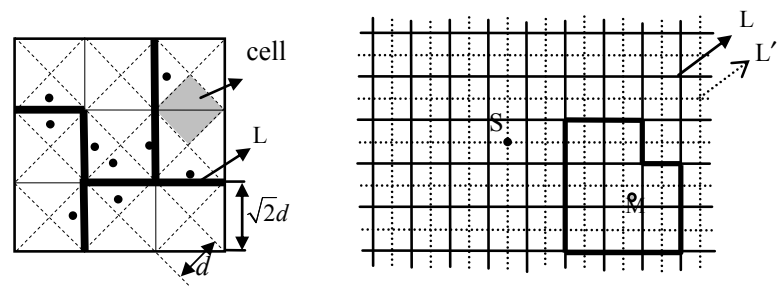

Fig2. (a)percolation model

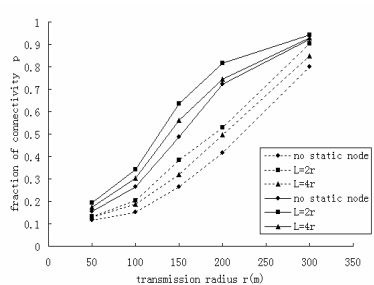

(b)lattice $L_{\text {and its dual lattice } L^{\prime}}$

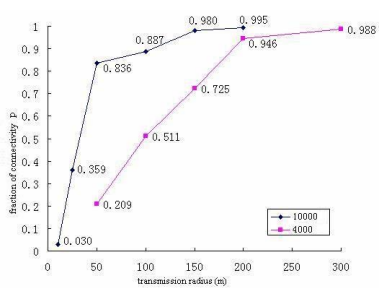

Fig3. (a)No. 312 National Road (b)The region of Wujiaochang

real 1-D ad hoc networks when the transmission radius is large enough, and static nodes are benefit for the improvement of connectivity, where real lines denote $\lambda=0.1$ and dashed lines denote $\lambda=0.05$, i.e. the total number of vehicles on the road is 1600 and 800 , respectively. Fig.3(b) shows with the increase of transmission radius or density of vehicles, the connectivity ratio $p$ increases, especially when the density is larger, the curve changes abrupt. A little increase of radius can reach high ratio of connectivity. We also simulate the point-based coverage, choosing the center of Wujiaochang as the given point to test the area covered by the point. The results of these tests show that due to the limit of the number of mobile nodes, the percolation cannot occur in real 2-D wireless network, however, with certain density, little change of radius induces a great shift of connectivity.

\section{ACKNOWLEDGMENTS}

The work presented in this paper was supported by the National Natural Science Foundation Grant No.90612006 ;

\section{REFERENCES}

[1] Dousse O, Baccelli F and Thiran P. Impact of interferences on connectivity in ad hoc networks. IEEE/ACM Transactions on networking, vol. 13, no. 2, April 2005.pp425-436.

[2] Benyuan Liu, Peter Brass, Olivier Dousse. Mobility Improves Coverage of Sensor Networks. MobiHoc'05, May 25-27, 2005, UrbanaChampaign, Illinois, USA

[3] J. van den Berg, Ronald Meester, Damien G. White. Dynamic Boolean models. ELSEVIER. Stochastic Processes and their Applications 69 (1997) pp247-257 